\title{
Prognostic Value of Bone Markers in Patients with Carcinoma
}

Nicole Zulauf', Marcus D Speda', Gerhard M Oremek ${ }^{1 *}$ and Ingo Marzi ${ }^{2}$

${ }^{1}$ Department of Laboratory Medicine Johann Wolfgang Goethe University, Germany

${ }^{2}$ Department of Surgery Johann Wolfgang Goethe University, Germany

\begin{abstract}
Background: We investigated the usefulness of bone markers, respectively Alkaline Phosphatase (AP) and Tartrat Resistant Acid Phosphatase 5b (TRACP 5b) for diagnosis, treatment and monitoring of patients with carcinoma of different origin. AP is a marker of bone formation, while TRACP $5 \mathrm{~b}$ is a marker of bone resorption. The isoform $5 \mathrm{~b}$ of the enzyme TRACP is expressed by osteoclasts and can be measured in blood.

Objectives: The aim of this study was to evaluate the prognostic value of the bone markers AP and TRACP $5 b$ to detect bone metastasis and pathological bone metabolism.

Materials and Methods: Our study comprised 101 patients with positive tumor markers. Sera of these patients were collected and the bone markers AP and TRACP $5 b$ were determind. TRACP $5 b$ was measured by a colorimetric test which determines the TRACP $5 b$ by using the phophatase activity of this enzyme by dephosphorylation of p-Nitrophenylphosphate (pNPP). The test is a two site immunoassay by Medac Diagnostika, Germany.

Results: The sensitivity of AP in our study to detect bone metastasis is $52.9 \%$, the specificity is $53.9 \%$. TRACP $5 \mathrm{~b}$ shows a sensitivity of $64.7 \%$ and a specificity of $70.9 \%$. An elevated TRACP $5 \mathrm{~b}$ activity is associated significantly with bone metastasis in our study groups $(p=0.01)$. In patients with chronic elevated levels of liver enzymes we could see a significant elevation of TRACP $5 b(p=0.005)$.

Conclusion: In conclusion TRACP $5 \mathrm{~b}$ is more sensitive and specific to detect bone metastasis and bone turnover than the Alkaline Phosphatase. In patients with multimorbidity the origin of AP is not clear due to its multiorganic appearance. The levels of TRACP $5 b$ are elevated in patients with bone metastasis and in patients with chronical dysfunction of the liver. TRACP $5 \mathrm{~b}$ might be helpful in the diagnostic procedure of tumor-patients to detect bone metastasis. Moreover TRACP $5 \mathrm{~b}$ seems to be helpful to indicate oncological patients with early dysfunctions in bone metabolism and helps to induce early treatment to these patients.
\end{abstract}

Keywords: Bone marker; Tumor marker; Bone metastasis; Malignancy; Alkaline Phosphatase (AP); Tartrate Resitant Acid Phosphatase (TRACP 5b)

\section{Introduction}

Malignant diseases and metastasis induced by those diseases are topics of medical research through decades. Bone is a common site for metastasis and is often elevating pain, morbidity and mortality. Through the past years several laboratory methods and markers have been developed to assist the diagnosis of bone lesions.

The accurate diagnostic of bone metastasis and the early induced antiresorptive and cancer therapy are essential for reducing morbidity and mortality in those patients and hence an important task.

Bone is a tissue which underlies continuous resorption and formation. Malignant tumors which are spreading to bone are causing osteolytic, osteoblastic or mixed lesions. Bone is resorbed by osteoclasts rather than through the tumor cells itselves [1,2].

Under these circumstances bone turnover is elevated and some of the involved enzymes and metabolic products can be measured. Several markers of bone turnover with different sensitivity and specificity have been described so far.

The Alkaline Phosphatase is one marker of bone formation which is often used due to its wide availability as a laboratory parameter [3]. It is a membrane-bound enzyme which can be found in diverse tissues of the body, such as bone, liver, kidney and the intestine. The exact function in bone metabolism is unkown, but AP increases when mineralization of bone is in progress [4].

In a healthy adult $50 \%$ of the total amount of AP are of liver origin and $50 \%$ of bone origin. In children and growing infants up to $90 \%$ of the total amount are of bone origin due to the higher expansive bone metabolism. Is a liver disease ruled out, AP provides an impression of the osteoblastic activity [5-7].

The TRACP is an enzyme expressed in bone resorbing osteoclasts, certain tissue macrophages and alveolar macrophages of the lung. Five isoforms of TRACP have been identified so far [8]. The two isoforms identified in the circulation have been named TRACP $5 \mathrm{a}$ and $5 \mathrm{~b}$. A correlation between TRACP $5 \mathrm{~b}$ and other markers of bone turnover or bone mineral density has been verified, but not for TRACP 5a [9]. TRACP $5 b$ is the osteoclastic specific isoform of the Tartrat Resistant Acid Phosphatase, also called Purple Acid Phosphatase due to its colour in purified form. Its usefulness as a marker of bone turnover has been described earlier [10].

Although these markers have a potential as tools in the diagnosis of bone metastasis, further analysis of these markers has been recommended to confirm the prediction of diagnosing bone turnover

*Corresponding author: Gerhard M Oremek, Department of Laboratory Medicine Internal Medicine, Hospital of the Johann Wolfgang Goethe University, Theodor Stern Kai 7, D- 60590 Frankfurt/ Main, Germany, Tel: +49(0)69/ 6301-5024; 7823; E-mail: Gerhard-Maximilian.Oremek@kgu.de

Received November 27, 2013; Accepted December 24, 2013; Published December 26, 2013

Citation: Zulauf N, Speda MD, Oremek GM, Marzi I (2013) Prognostic Value of Bone Markers in Patients with Carcinoma. J Mol Biomark Diagn 5: 159 doi:10.4172/2155-9929.1000159

Copyright: @ 2013 Zulauf N, et al. This is an open-access article distributed under the terms of the Creative Commons Attribution License, which permits unrestricted use, distribution, and reproduction in any medium, provided the original author and source are credited 
and monitoring antiresorptive treatment. The aim of this study was to evaluate the clinical value of AP and TRACP $5 \mathrm{~b}$ to detect bone metastasis and pathological bone metabolism in a collective of patients with positive tumor markers.

\section{Materials and Methods}

Peripheral blood samples of 101 patients with positive tumor markers have been collected. A total number of 19 patients had no malignant disease. 60 patients were suffering from a malignant disease with known metastasis but no bone metastasis. 17 patients had known bone metastasis.

Among the patients with malignant cancer diseases a total number of 11 patients had ovarial carcinoma, 14 colon carcinoma, 25 patients carcinoma of the breast, 6 pancreatic carcinoma, 8 patients carcinoma

\begin{tabular}{|c|c|c|c|c|c|c|c|}
\hline & \multicolumn{3}{|c|}{ TRACP 5b [U/I] } & \multicolumn{3}{c|}{ AP [U/I] } \\
\cline { 2 - 8 } & N & Mean & $\begin{array}{c}\text { Std. } \\
\text { Dev. }\end{array}$ & Range & Mean & $\begin{array}{c}\text { Std. } \\
\text { Dev. }\end{array}$ & Range \\
\hline $\begin{array}{c}\text { Patients without } \\
\text { malignant disease }\end{array}$ & 19 & 4.22 & 2.37 & $1.87-10$ & 171.37 & 114.11 & $59-428$ \\
\hline $\begin{array}{c}\text { Patients with known } \\
\text { malignant disease } \\
\text { without bone metastasis }\end{array}$ & 60 & 5.04 & 2.12 & $1.71-10$ & 262.80 & 219.47 & $69-1045$ \\
\hline $\begin{array}{c}\text { Patients with known bone } \\
\text { metastasis }\end{array}$ & 17 & 6.75 & 3.0 & $2.23-10$ & 495.41 & 712.51 & $105-3053$ \\
\hline
\end{tabular}

Table 1: Activity of TRACP $5 b$ and AP [U/l] in our different study groups.

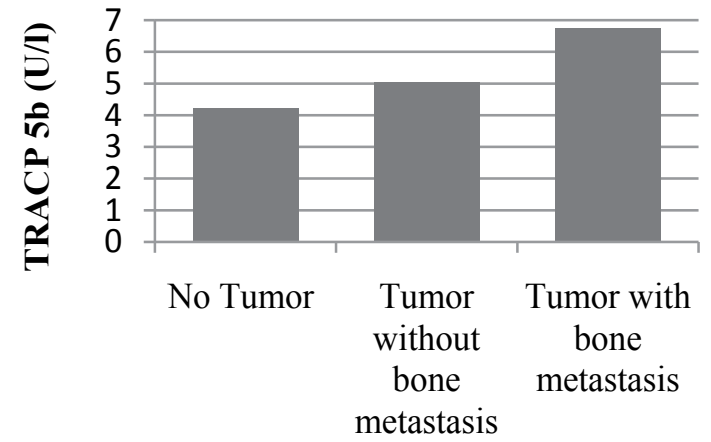

Figure 1: Distribution of mean TRACP $5 b$ in our study. Activity of TRACP $5 \mathrm{~b}(\mathrm{U} / \mathrm{l})$ against patients without a malignant disease, patients with known malignancy but without bone metastasis, patients with malignancy and bone metastasis.

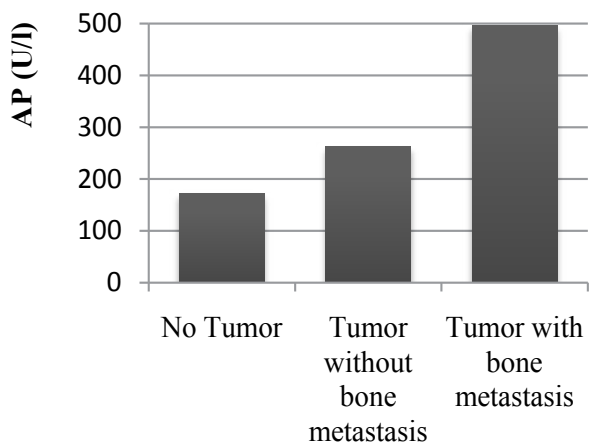

Figure 2: Distribution of mean AP in our study. Activity of $A P(U / I)$ against patients without a malignant disease, patients with known malignancy but without bone metastasis, patients with malignancy and bone metastasis. of the liver, 4 patients neuroblastoma and 9 patients with carcinoma of other origin. In our collective 5 patients were under the age of 10 , all of these patients had a TRACP $5 \mathrm{~b}$ acticity of $10 \mathrm{U} / \mathrm{l}$. This inverse correlation between age and TRACP $5 b$ activity has been described earlier and these patients have been cancelled for further investigation due to the cause of expansive bone turnover in children.

The analysis of Alkaline Phosphatase has been conducted by using the Hitachi 917 Analyzer. The determination of TRACP 5b has been done by a two site immunoassay (Medac Diagnostika GmbH, Germany) with two specific monoclonal antibodies, which have been proved not to crossreact with the isoform TRACP $5 \mathrm{a}$. Incubating the sera samples with p-Nitrophenylphosphate (pNPP) leads to a dephosphorylation of this agent. The product of this reaction could be measured photometrically. The colorimetric measurement to determine the TRACP $5 \mathrm{~b}$ activity has been performed with the SLT Spectra Photometer (SLT Instruments $\mathrm{GmbH}$, Austria).

\section{Statistical Analysis}

All the statistical calculations were performed with SPSS Statistics 21.0 and Microsoft Excel 2007.

\section{Results}

The results of AP and TRACP 5b activity in our different study groups are summarized in Table 1 . The mean activity of AP in the group without malignancy has been $171.37 \mathrm{U} / \mathrm{l}$, in patients with malignant disease but without bone metastasis $262.80 \mathrm{U} / \mathrm{l}$ and in patients with bone metastasis $495.41 \mathrm{U} / \mathrm{l}$.

The serum levels of TRACP $5 \mathrm{~b}$ activity in patients without malignancy showed a mean of $4.22 \mathrm{U} / \mathrm{l}$. The highest activities have been found in patients with malignancy and additional bone metastasis (mean $6.75 \mathrm{U} / \mathrm{l}$ ) and in patients with malignancy (mean activity of 5.04 $\mathrm{U} / \mathrm{l})$.

A significant difference between the patients without malignant disease, those with malignancy and the patients with additional bone metastasis could be shown for AP (Kruskal: $p=0.009$ ) and TRACP $5 b$ (Kruskal: $\mathrm{p}=0.01$ ).

The distribution of AP and TRACP $5 \mathrm{~b}$ in our patients is shown in Figure 1 and 2.

Figure 3 and 4 are showing the categorised results of AP and TRACP $5 \mathrm{~b}$ whether they were normal or above normal activity. For the AP we could not figure out a significant difference of AP in our study groups (Chi-square test: 0.49). We found a significant association between the occurancy of bone metastasis and elevated TRACP $5 \mathrm{~b}$ activity in our study (Chi-square test: 0,01). In our study the sensitivity of AP to detect bone metastasis is $52.9 \%$ and for TRACP $5 \mathrm{~b} 64.7 \%$. The specificity for AP has been $53.9 \%$ and for TRACP $5 \mathrm{~b} 70.9 \%$.

\section{Discussion}

In the last years several different bone markers have been investigated to assist the diagnosis of bone turnover. However the accuracy of those markers does not allow to be the only diagnostic instrument to predict the appearance of bone metastasis. So far none of the known markers could be solely used for a screening of patients to diagnose metastasis to bone.

The measured sensitivity and specificity for AP and TRACP $5 b$ to detect bone metastasis in our study is contrary to our expectations lower than results of earlier investigations, which is mainly due to our randomised selection of patients with positive tumor markers $[11,12]$. 


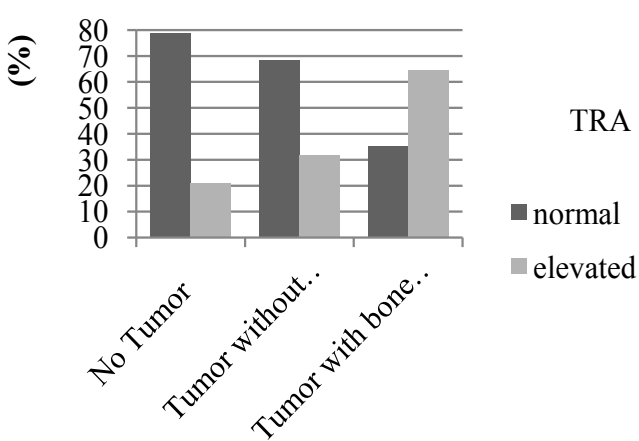

Figure 3: Distribution of TRACP $5 \mathrm{~b}$ in our study groups. In patients without tumor $78.9 \%$ are of normal activity, $21.1 \%$ are pathological elevated. Patients with tumor but without bone metastasis show normal activity in $68.3 \%$ and elevated levels of TRACP $5 \mathrm{~b}$ in $31.7 \%$. The TRACP $5 \mathrm{~b}$ levels were normal in $35.3 \%$ of all patients with known bone metastasis and elevated in $64.7 \%$.

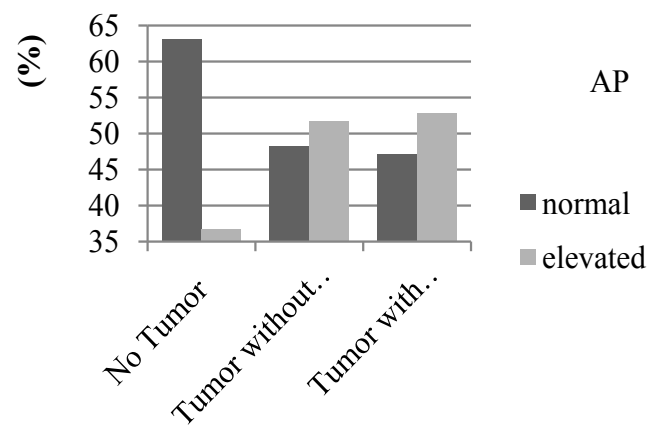

Figure 4: Distribution of AP in our study groups. In patients without tumor 63.2 $\%$ are of normal activity, $36.8 \%$ are pathological elevated. Patients with tumor but without bone metastasis show normal activity in $48.3 \%$ and elevated levels of AP in $51.7 \%$. The AP levels were normal in $47.1 \%$ of all patients with known bone metastasis and elevated in $52.9 \%$.

The poor sensitivity and specificity for AP appeared to be caused by the multimorbidity of our patients. In those patients the elevated AP levels seemed to be caused by an origin different than bone. At least $35.3 \%$ of our patients with known malignancy and bone metastasis did not show pathological elevated serum levels of TRACP 5b, at least 9 patients of that group had been treated with bisphosphonates at the time of investigation [13]. $21.1 \%$ of the patients with no known malignancy showed elevated serum levels of TRACP $5 b ; 3$ of these patients were pregnant and 1 patient was suffering from a cirrhosis of the liver of unknown genesis. An increasing bone turnover in pregnancy has been described previous [14]. The coincidence of a decreased bone density and liver diseases has also been formerly analyzed [15-17]. As investigated before, we found a slender $(\mathrm{r} \leq 0.5)$ but high significant correlation between our measurements of AP and TRACP 5b [18]. In comparison to AP, the TRACP $5 \mathrm{~b}$ has been the more specific bone marker to detect bone metastasis in our study.

A significant correlation between the activity of TRACP $5 b$ and the occurancy of bone metastasis has been statistically proved (Kruskal: $\mathrm{p} \leq 0.01$, Multivariat analysis: $\mathrm{p} \leq 0.05$, Chi-square test: $\mathrm{p} \leq 0.05$ ). Furthermore TRACP $5 \mathrm{~b}$ showed in our study a significant increase in patients with chronical elevated liver enzyms (Mann-Whitney-U test: $p$ $\leq 0.05$ ). It is known that a chronical pathological effected liver disease affects the bone metabolism and osteoporosis occurs more often. We suggest a further investigation and evaluation of TRACP $5 \mathrm{~b}$ as a marker of bone turnover. It seems to be a helpful diagnostic parameter of pathological elevated bone metabolism and should be used in follow up examinations of oncological patients. It seems to be not only a helpful tool to get an idea of the bone metabolism, but may be also helpful to assist an antiresorptive treatment in time to those patients with progressive affection of the bone caused by chronic liver disease.

\section{References}

1. Boyce BF, Yoneda T, Guise TA (1999) Factors regulating the growth of metastatic cancer in bone. Endocr Relat Cancer 6: 333-347.

2. Mundy GR, Martin TJ (1993) Pathophysiology of skeletal complications of cancer. Physiology and Pharmacology of Bone, Springer: 641-671.

3. Oremek GM, Weis A, Sapoutzis N, Sauer-Eppel H (2003) Diagnostic value of bone and tumour markers in patients with malignant diseases. Anticancer Res 23: $987-990$

4. Lian JB, Stein GS, Aubin JE (2003) Anatomy and Biology of Bone Matrix and Cellular Elements. Bone Formation: Maturation and Functional Activities of Osteoblast Lineage Cells. American Society for Bone and Mineral Research, Primer: 13-27.

5. Fohr B, Dunstan CR, Seibel MJ (2003) Clinical review 165: Markers of bone remodeling in metastatic bone disease. J Clin Endocrinol Metab 88: 5059-5075.

6. Woitge HW, Seibel MJ (2001) Biochemical markers to survey bone turnover Rheum Dis Clin North Am 27: 49-80.

7. Mose S, Menzel C, Kurth AA, Obert K, Breidert I, et al. (2003) Tartrate-resistant acid phosphatase $5 \mathrm{~b}$ as serum marker of bone metabolism in cancer patients. Anticancer Res 23: 2783-2788.

8. Yam LT, Janckila AJ (2003) Tartrate-resistant acid phosphatase (TRACP): a personal perspective. J Bone Miner Res 18: 1894-1896.

9. Halleen JM, Alatalo SL, Suominen H, Cheng S, Janckila AJ, et al. (2000) Tartrate-resistant acid phosphatase $5 \mathrm{~b}$ : a novel serum marker of bone resorption. J Bone Miner Res 15: 1337-1345.

10. Halleen J, Hentunen TA, Hellman J, Väänänen HK (1996) Tartrate-resistan acid phosphatase from human bone: purification and development of an immunoassay. J Bone Miner Res 11: 1444-1452.

11. Halleen JM, Alatalo SL, Janckila AJ, Woitge HW, Seibel MJ, et al. (2001) Serum tartrate-resistant acid phosphatase $5 \mathrm{~b}$ is a specific and sensitive marker of bone resorption. Clin Chem 47: 597-600.

12. Seregni E, Martinetti A, Ferrari L, Bombardieri E (2001) Clinical utility of biochemical marker of bone remodelling in patients with bone metastases of solid tumors. Q J Nucl Med 45: 7-17.

13. Terpos E, Viniou N, de la Fuente J, Meletis J, Voskaridou E, et al. (2003) Pamidronate is superior to ibandronate in decreasing bone resorption, interleukin-6 and beta 2-microglobulin in multiple myeloma. Eur J Haematol 70: $34-42$

14. Karlsson MK, Ahlborg HG, Karlsson C (2005) Maternity and bone mineral density. Acta Orthop 76: 2-13.

15. Collier JD, Ninkovic M, Compston JE (2002) Guidelines on the management of osteoporosis associated with chronic liver disease. Gut 50 Suppl 1: i1-9.

16. Floreani A, Mega A, Camozzi V, Baldo V, Plebani M, et al. (2005) Is osteoporosis a peculiar association with primary biliary cirrhosis? World J Gastroenterol 11: 5347-5350.

17. Schiefke I, Fach A, Wiedmann M, Aretin AV, Schenker E, et al. (2005) Reduced bone mineral density and altered bone turnover markers in patients with noncirrhotic chronic hepatitis B or C infection. World J Gastroenterol 11: 1843-1847.

18. Koizumi M, Takahashi S, Ogata E (2003) Bone metabolic markers in bisphosphonate therapy for skeletal metastases in patients with breast cancer. Breast Cancer 10: 21-27. 